\title{
EPS Production, PHB Accumulation and Abiotic Stress Endurance of Plant Growth Promoting Methylobacterium Strains Grown in a High Carbon Concentration
}

\author{
Sungman Woo, Parthiban Subramanian, Krishnamoorthy Ramasamy, \\ M. Melvin Joe, and Tongmin Sa* \\ Department of Environmental and Biological Chemistry, Chungbuk National University, \\ Cheongju, 361-763, Korea
}

\begin{abstract}
In this study, we compared growth pattem, floc yield, Exo-polysaccharides (EPS) production, Poly- $\beta$ -hydroxybutyrate (PHB) accumulation, resistance to osmotic and acid stress in Methylobacterium strains CBMB20, CBMB27, CBMB35, and CBMB110. Modified high C:N ratio medium denoted as HCN-AMS medium was used with a $\mathrm{C}: \mathrm{N}$ ratio of 30:1. The HCN-AMS medium favored increased growth in all the studied strains. All Methylobacterium strains tested positive for EPS production and showed positive fluorescence with calcoflour stain. Elevated levels of EPS production from 4.2 to $\mathbf{7 5 . 0 \%}$ was observed in HCN-AMS medium. Accumulation of PHB in HCN-AMS medium increased by 3.8, 36.7, and $12.0 \%$ in strains CBMB27, CBMB35, and CBMB110 respectively. Among the abiotic stresses, osmotic stress-induced growth inhibition of Methylobacterium strains was found to be lowered when grown in HCN-AMS medium. Likewise, growth inhibition due to acid stress at pH 5.0 was lower for strains grown in HCN-AMS medium compared to growth in AMS medium. Enhanced survivability understress conditions may be attributed to the high EPS and PHB production at increased carbon concentration in the growth medium.
\end{abstract}

Key words: Methylobacterium, AMS medium, HCN-AMS medium, EPS production, PHB accumulation, Stress endurance efficiency

\section{Introduction}

Members of the genus Methylobacterium, often referred to as pink-pigmented facultative methylotrophic bacteria (PPFMs) are distributed in soil, leaves and various other parts of plant (Corpe and Rheme, 1989; Green, 2001). These bacteria utilize methanol produced out of the stomata of plants, and are also able to grow on a variety of C2, C3, and C4 compounds (Corpe, 1985). Although PPFMs often dominate the phyllosphere region of plants (Corpe and Rheem, 1989), their presence in the rhizosphere has also been reported (Idris et al., 2004; Omer et al., 2004). They benefit the plants they colonize by production of indole acetic acid, cytokinins and vitamin $\mathrm{B}_{12}$ and through endophytic and endo-symbiotic relationships with plants (Omer et al., 2004; Knief et al., 2008). Their

\footnotetext{
Received : 2012. 7. 17 Accepted : 2012. 8. 16

*Corresponding author : Phone: +82432612561

E-mail: tomsa@chungbuk.ac.kr
}

association with more than 70 plant species makes them potential agents for plant growth promotion and bio control against diseases (Holland and Polacco, 1994).

In spite of their studied plant growth promotional traits, commericalization of the Methylobacterium strains as bioinoculant have been hindered constantly owing to lack of appropriate formulation. Inoculant formulation is important in providing a suitable microenvironment during storage in different carrier material and in preventing rapid decline of introduced bacteria in the soil (Bashan, 1998). Van Elsas and Heijnen (1990) suggested that formulations lacking these characteristic features will be unacceptable in the agricultural market.

The physiological status of microorganisms in a bioinoculant formulation is considered more important than their cell numbers to ensure higher survival in carriers, soil and seed Catroux et al. (2001). One such approach for inoculant preparation is based on the induction of massive cell aggregation, clumping and flocculation (Sadasivan and Neyra, 1985). Neyra et al. (1995) produced inoculants 
containing a mixture, of both Azospirillum and Rhizobium, by coflocculation forming intergeneric coaggregates. They also reported that these cells can be grown on a large scale and used as inoculants in agriculture.

Although aggregation/flocculation has been reported among various Methylobacterium spp. such as Methylobacterium organophilum (Choi, 1998), Methylobacterium adhaesivum AR27 (Gallego et al. 2006), and Methylobacterium marchantiae JT1 (Schauer et al., 2011), this phenomenon has never been studied extensively among Methylobacterium spp. Moreover, no attempt to the best of our knowledge has been made to develop Methylobacterium aggregated cells as bioinoculants for agriculture.

The present study evaluated Methylobacterium strains CBMB20, CBMB27, CBMB35, and CBMB110 for their growth pattern, floc yield, Exo-polysaccharides (EPS) production, Poly- $\beta$-hydroxybutyrate (PHB) accumulation and stress endurance efficiency. Ammoniun mineral salt (AMS) medium is a selective media used to grown and maintain Methylobacterium strains (Madhayan, 2007). In the present study AMS medium was modified by increasing carbon concentration to attain a C: $\mathrm{N}$ ratio of $30: 1$.

\section{Materials and Methods}

\section{Studies on culture conditions for maximizing} aggregation Methylobacterium strains grown in ammonium mineral salt (AMS) media with $0.5 \%$ sodium succinate were transferred to $25 \mathrm{~mL}$ AMS broth and incubated at $30^{\circ} \mathrm{C}$ on a shaker incubator $(150 \mathrm{rpm})$ for 72 h. After $72 \mathrm{~h}$ incubation, $1.0 \mathrm{~mL}$ of culture $\left(1 \times 10^{8} \mathrm{cfu}\right.$ $\mathrm{mL}^{-1}$ ) was transferred to $100 \mathrm{~mL}$ fresh AMS broth and the culture was allowed to grow for another $72 \mathrm{~h}$. To obtain a maximum aggregation/flocculation and for enhanced accumulation of EPS and PHB, AMS medium was modified by increasing the carbon concentration. The carbon source of AMS, sodium succinate concentration was increased to $1.1 \%$ and the final $\mathrm{C}: \mathrm{N}$ ratio in the media was set to 30:1. This modified high $\mathrm{C}: \mathrm{N}$ ratio medium was denoted as HCN-AMS medium elsewhere in the text. The composition of AMS media is given in Table 1.

Quantification of the flocculation The flocs were harvested on the $3^{\text {rd }}$ day of incubation by filtering the flocs through whatman filter paper (No. 1). For dry weight determination, the filter paper with the flocs were placed
Table 1. Composition of ammonium mineral salt (AMS) medium.

\begin{tabular}{lc}
\hline \hline Chemicals & Amount per liter \\
\hline $\mathrm{NH}_{4} \mathrm{Cl}$ & $0.50 \mathrm{~g}$ \\
$\mathrm{~K}_{2} \mathrm{HPO}_{4}$ & $0.70 \mathrm{~g}$ \\
$\mathrm{KH}_{2} \mathrm{PO}_{4}$ & $0.54 \mathrm{~g}$ \\
$\mathrm{MgSO}_{4} \cdot 7 \mathrm{H}_{2} \mathrm{O}$ & $1.00 \mathrm{~g}$ \\
$\mathrm{CaCl}_{2} \cdot 2 \mathrm{H}_{2} \mathrm{O}$ & $0.200 \mathrm{~g}$ \\
$\mathrm{FeSO}_{4} \cdot 7 \mathrm{H}_{2} \mathrm{O}$ & $4.00 \mathrm{mg}$ \\
$\mathrm{ZnSO}_{4} \cdot 7 \mathrm{H}_{2} \mathrm{O}$ & $100.00 \mu \mathrm{g}$ \\
$\mathrm{MnCl}_{2} \cdot 4 \mathrm{H}_{2} \mathrm{O}$ & $30.00 \mu \mathrm{g}$ \\
$\mathrm{H}_{3} \mathrm{BO}_{3}$ & $300.00 \mu \mathrm{g}$ \\
$\mathrm{CoCl}_{2} \cdot 6 \mathrm{H}_{2} \mathrm{O}$ & $200.00 \mu \mathrm{g}$ \\
$\mathrm{CuCl}_{2} \cdot 2 \mathrm{H}_{2} \mathrm{O}$ & $10.00 \mu \mathrm{g}$ \\
$\mathrm{NiCl}_{2} \cdot 6 \mathrm{H}_{2} \mathrm{O}$ & $20.00 \mu \mathrm{g}$ \\
$\mathrm{Na}_{2} \mathrm{MoO}_{4} \cdot 2 \mathrm{H}_{2} \mathrm{O}$ & $60.00 \mu \mathrm{g}$ \\
$\mathrm{Sodium} \mathrm{succinate}$ & $5.00 \mathrm{~g}$ \\
$\mathrm{pH}$ & 6.80 \\
\hline
\end{tabular}

In HCN-AMS medium concentration of sodium succinate $11.0 \mathrm{~g}$ and $\mathrm{NH}_{4} \mathrm{Cl}$ concentration to $0.25 \mathrm{~g}$.

in a desiccator oven at $60^{\circ} \mathrm{C}$ for $2 \mathrm{~h}$ and weighed. The weight of the filter paper was then excluded and floc yield was expressed as mg per liter of medium $\left(\mathrm{mg} \mathrm{L}^{-1}\right)$.

\section{Qualitative/ Quantitative assay for EPS production}

Qualitative estimation of EPS production Plates containing Calcofluor white were used to qualitatively examine EPS production (Dana and Shimkets, 1993; Ramaswamy et al., 1997). Briefly, $5 \mu \mathrm{L}$ of a cell suspension at $5 \times 10^{9}$ cells $\mathrm{mL}^{-1}$ was spotted onto CYE plates supplemented with Calcofluor white $\left(50 \mu \mathrm{g} \mathrm{mL}^{-1}\right)$. These plates were incubated at $32^{\circ} \mathrm{C}$ for 5 days before documentation. Calcofluor white plates were examined under long-wave UV (365 nm) for fluorescence, which is indicative of dye binding and EPS production.

\section{Quantitative estimation of EPS production Bacterial} EPS content was estimated according to the protocol of Celik et al. (2008). Bacterial strains grown in the HCN-AMS medium were harvested by centrifugation $(5000 \times g$, washed with saline $0.9 \% \mathrm{NaCl} \mathrm{w} / \mathrm{v})$ and the resulting pellets were stirred with glass beads to detach EPS associated with the bacterial cells. The supernatant 
was then removed by centrifugation $(10,000 \times g$ for 30 min, $4^{\circ} \mathrm{C}$ ) and to the pellet, six volumes of $95 \%$ ethanol was added and allowed to precipitate over night at $4{ }^{\circ} \mathrm{C}$. Precipitated EPS were recovered by centrifugation $\left(12,000 \times g\right.$ for $\left.30 \mathrm{~min}, 4^{\circ} \mathrm{C}\right)$, and the resulting pellets were dissolved in distilled water. Total EPS content was estimated by phenol-sulphuric method using glucose as standard.

\section{Qualitative/Quantitative assay for PHB production}

Qualitative estimation of PHB Two microscopic techniques were used to detect PHB producing bacteria. Phase contrast microscopy detects brightly refractile cytoplasmic inclusions (RCI) in axenic cultures of PHB producing bacteria (López-Cortés et al., 2008). The lipophilic stain, Sudan Black was used to identify PHB under bright field and epifluorescence microscopes, respectively. Sudan Black staining was performed on heat-fixed samples and the staining solution was prepared by dissolving $0.3 \mathrm{~g}$ Sudan Black B in $75 \mathrm{~mL}$ of $95 \%$ ethanol, and the volume brought to $100 \mathrm{~mL}$ with distilled water. Samples were stained with Sudan Black solution for $10 \mathrm{~min}$, blot dried with filter paper, and clarified with xylene drops, dried again with filter paper, and counter stained with $0.5 \%$ aqueous safranine for $5 \mathrm{~s}$ (López-Cortés, 2008).

Quantitative estimation of PHB Bacterial cell cultures grown HCN-AMS medium were harvested and the pellets were resuspended in sodium hypochloride, of the same original volume of medium. After $1 \mathrm{~h}$ incubation at $37^{\circ} \mathrm{C}$, the lipid granules (PHB) were centrifuged, washed with distilled water, and subsequently washed with acetone and ethanol. The polymer of PHB was dissolved by extraction with three small portions of boiling chloroform; the chloroform solution was filtered, and the filtrate was used. The excess of chloroform was evaporated, and 10 $\mathrm{mL}$ of concentrated $\mathrm{H}_{2} \mathrm{SO}_{4}$ was added and heated for 10 min at $100^{\circ} \mathrm{C}$ in a boiling water bath to convert PHB to crotonic acid. PHB was determined as crotonic acid and quantified by spectrophotometer at $235 \mathrm{~nm}$. A standard curve with commercial PHB (Sigma) was made to determine the PHB present in the samples (Karr et al., 1983; Reusch and Sadoff, 1983).

\section{Stress endurance efficiency under different induced} stress conditions Stress endurance experiments were carried out according to Kadouri et al. (2003) with a slight modification. For each experiment the initial number of bacterial cells was maintained higher than $9 \log \mathrm{cfu} \mathrm{mL}^{-1}$. The effect of osmotic stress was determined by exposing the bacterial cells to $150 \mathrm{mM}, 200 \mathrm{mM}$ and $250 \mathrm{mM} \mathrm{NaCl}$ for $30 \mathrm{~min}$ at $42^{\circ} \mathrm{C}$. The effect of acid stress was determined by exposing the bacterial cells to $330 \mathrm{mM}$ sodium lactate buffer at $\mathrm{pH} 5.0$ for $30 \mathrm{~min}$ at $42^{\circ} \mathrm{C}$. Serial dilution and plating was carried out after treartment with abiotic stress and plates were incubated for $48 \mathrm{~h}$ at $42^{\circ} \mathrm{C}$ under aerobic conditions.

Statistical analysis Data were analyzed in STATISTICA 7.0 following the protocols of Gomez and Gomez (1984) using $t$-test method. Critical Difference (CD) values were calculated at $P$ level of $0.05 \%$.

\section{Results}

Effect of HCN-AMS medium on Methylobacterium strains growth Growth of Methylobacterium strains in HCN-AMS medium was compared with growth in normal AMS medium without any modifications. In general, HCN-AMS medium favored a higher growth in all strains (Fig. 1). Increased growth was pronouncedly observed in CBMB27 strain, which recorded an OD value of 1.32 after $84 \mathrm{~h}$ incubation (Fig. 1b). Similarly, the strain CBMB110 also showed high OD value of 1.15 after $84 \mathrm{~h}$ incubation. However, in spite of marked differences during the 12 - 48 $\mathrm{h}$ incubation time, no differences were observed between CBMB35 strains grown in the two media after the $84 \mathrm{~h}$ incubation period (Fig. 1c).

\section{Effect of HCN-AMS medium on Methylobacterium}

population Population of Methylobacterium strains in conventional AMS medium and HCN-AMS medium was compared. In general, HCN-AMS medium increased the population in all the strains (Fig. 2). The biggest difference in growth between populations grown in the two media was seen in CBMB 110 with a $4.2 \%$ increase at $7.40 \mathrm{log}$ cfu $\mathrm{mL}^{-1}$ compared to CBMB110 grown in AMS medium. This was similarly followed by CBMB 35, CBMB 20, and CBMB 27 at 1.9, 0.9, and $0.4 \%$ growth increase in HCN-AMS medium compared to normal AMS medium. Except for that of CBMB 27, all differences were found to 


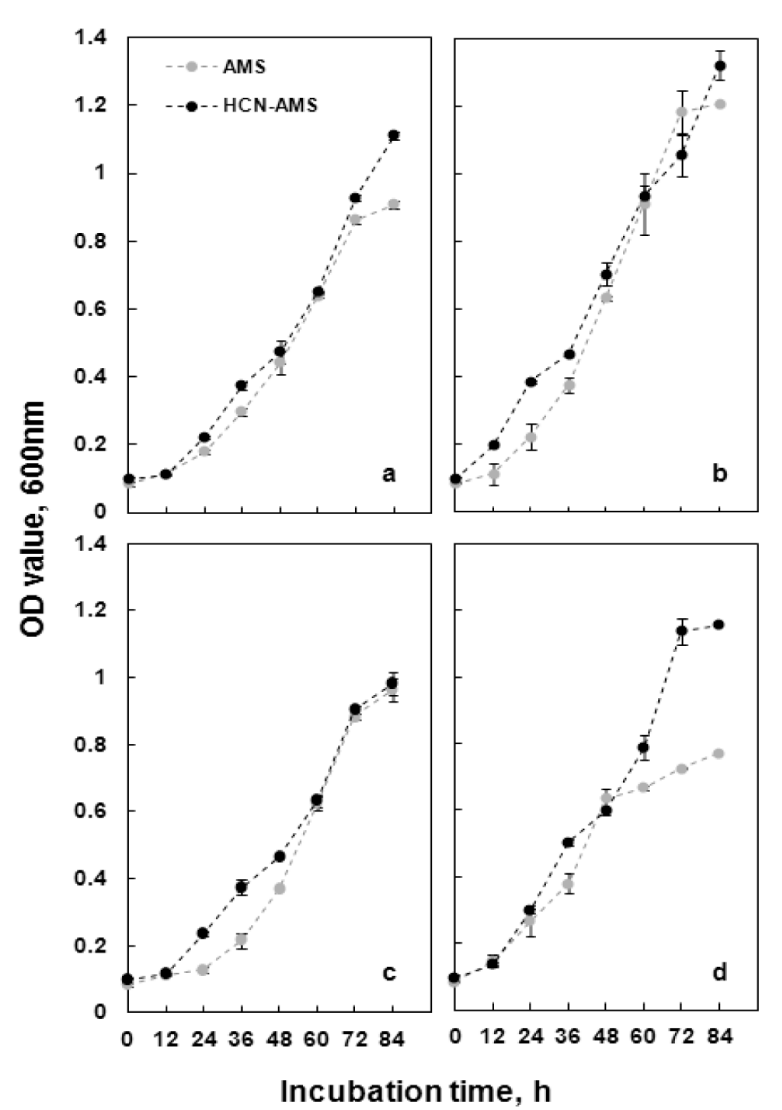

Fig. 1. Growth of Methylobacterium strains in AMS medium and HCN-AMS medium maintained at $150 \mathrm{rpm}$ at $30^{\circ} \mathrm{C}$. a) $\mathrm{CBMB20}$; b) $\mathrm{CBMB27}$; c) $\mathrm{CBMB35}$; d) CBMB110. Each value represents the mean $\pm \operatorname{S.E}(n=4)$.

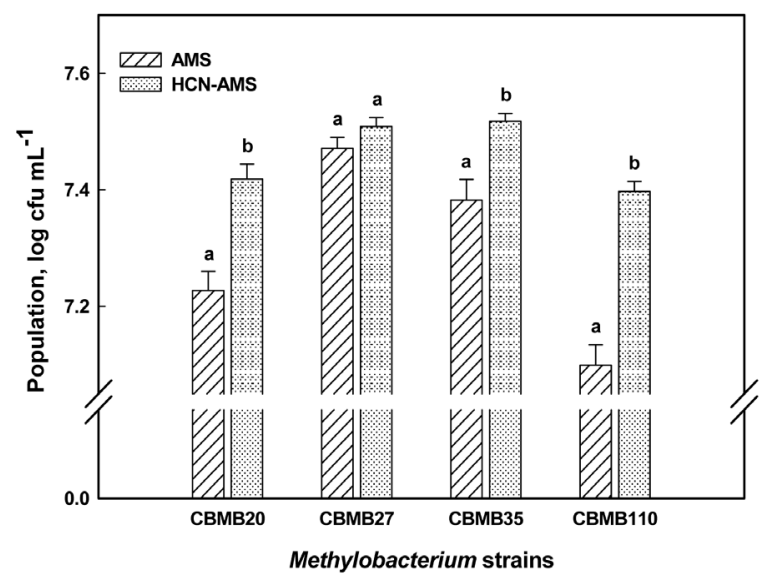

Fig. 2. Comparative population of Methylobacterium strains in AMS medium and HCN-AMS medium maintained at $150 \mathrm{rpm}$ at $30^{\circ} \mathrm{C}$. Each value represents the mean \pm S.E $(n=4)$. Different letters indicate a significant difference (between growth media) according to $t$ test at $p<0.05$.

be statistically significant.

Determination of flocculation yield Comparative floc yield of Methylobacterium strains in HCN-AMS

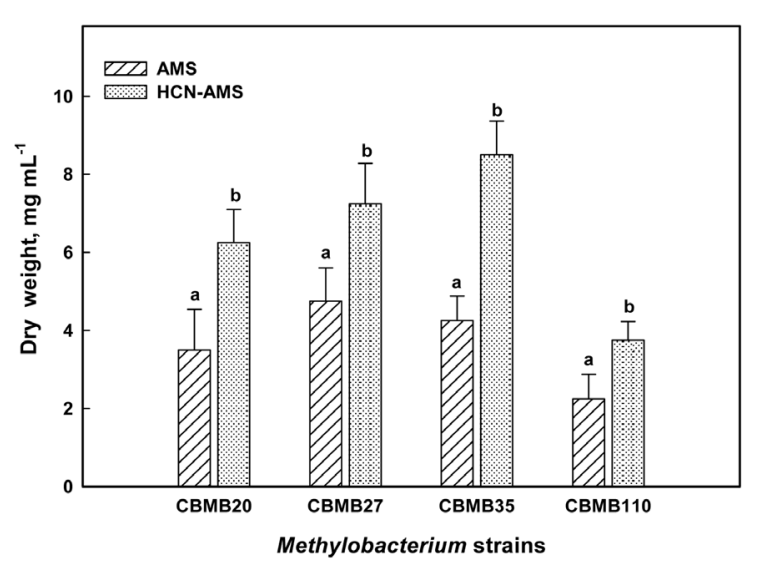

Fig. 3. Comparative flocculation yield of Methylobacterium strains in AMS medium and HCN-AMS medium maintained at $150 \mathrm{rpm}$ at $30^{\circ} \mathrm{C}$. Each value represents the mean $\pm \mathrm{SE}$ $(n=4)$. Different letters indicate significant difference (between growth media) according to $t$ test at $p \leq 0.05$.

medium and AMS medium were studied and the results are presented graphically in Fig. 3. Irrespective of the strain, a marked increase in the floc yield was recorded. Increases of 80.0, 52.1, 93.0, and $65.2 \%$ in floc yield was observed in CBMB20, CBMB27, CBMB35, and CBMB110 strains respectively, using HCN-AMS medium. Comparison in floc yield among the Methylobacterium strains grown in HCN-AMS medium revealed that CBMB35 produced the highest floc, followed by CBMB27 at 8.5 and $7.3 \mathrm{mg} \mathrm{mL}^{-1}$ dry weight, respectively.

Qualitative detemination of EPS production The calcofluor-binding polysaccharides were distributed between the capsular and exopolysaccharide fractions, suggesting their exocellular localization. Calcofluor-white is a special fluorescent stain that binds strongly to structures containing cellulose present in the polysaccharides. All Methylobacterium strains were found to be positive for calcoflour as evidenced by the positive fluorescence (Fig. 4). This positive staining provided evidence for the presence of neutral gleans in the polysaccharide fraction.

\section{Quantitative EPS production Quantitative EPS} production in Methylobacterium stains was studied based on a spectrometric assay in AMS medium and HCN-AMS medium. Though all the strains were able to produce EPS, the production efficiency in Methylobacterium stains varied considerably. Methylobacterium strains CBMB20, CBMB27, CBMB35, and CBMB110 recorded EPS contents of $0.34,0.23,0.25$, and $0.24 \mathrm{mg} \mathrm{mL}^{-1}$, respectively and 

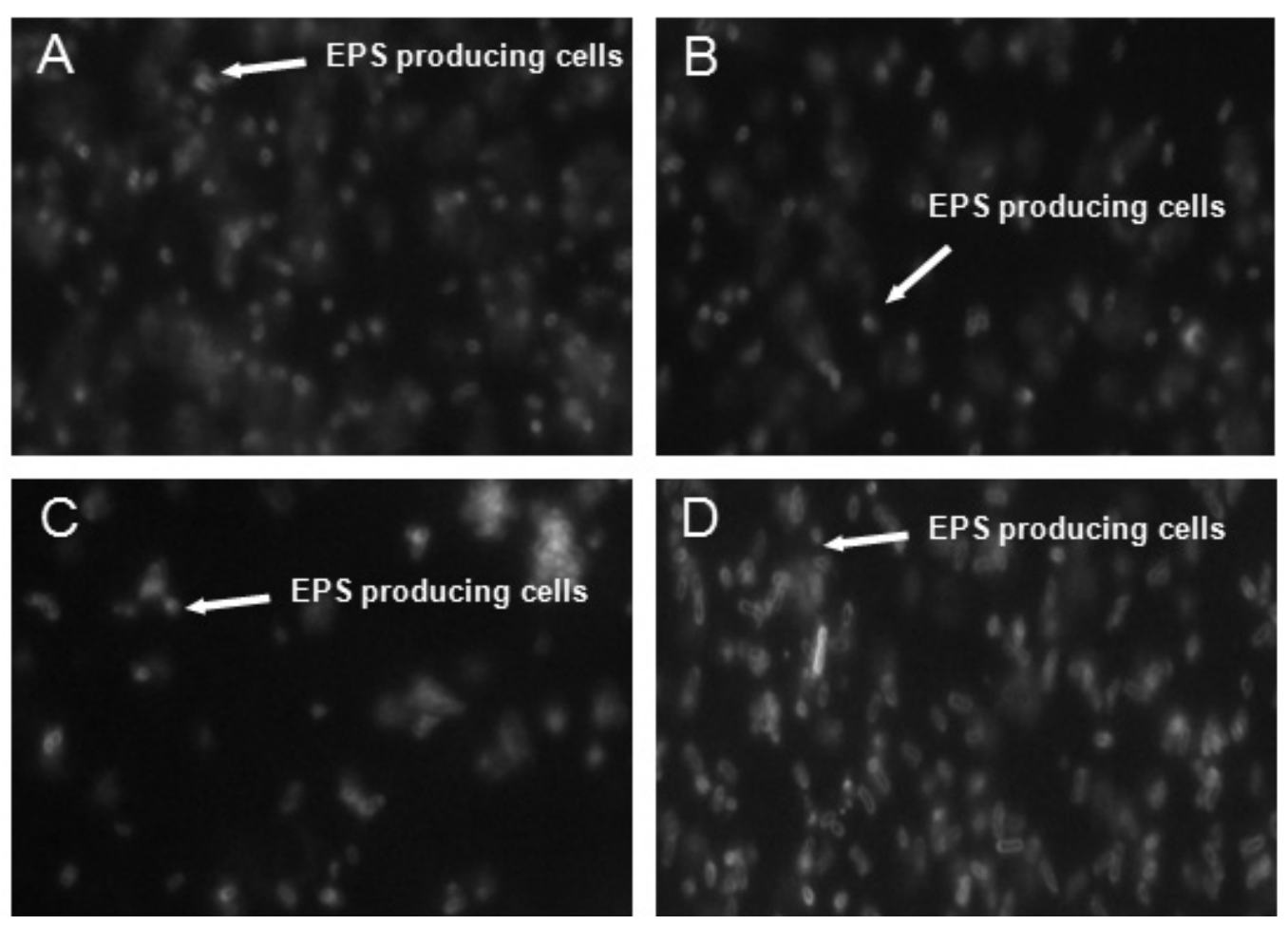

Fig. 4. Micrographs of Methylobacterium stains showing positive calcofluor florescence. Micrographed with microscope illuminator filter IF550 and DM dichronic mimor. A) CBMB20; B) CBMB27; C) CBMB35; D) CBMB110.

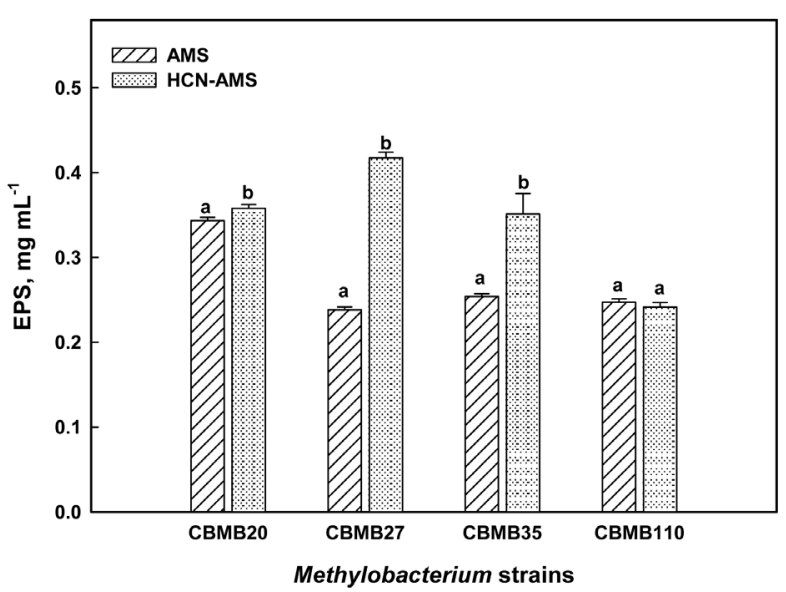

Fig. 5. EPS production of Methylobacterium strains grown in AMS medium and HCN-AMS medium maintained at 150 rpm at $30^{\circ} \mathrm{C}$. Each value represents the mean $\pm \operatorname{S.E}(n=4)$. Different letters indicate significant difference (between growth media) according to $t$ test at $p \leq 0.05$.

$0.35,0.41,0.35$, and $0.24 \mathrm{mg} \mathrm{mL}^{-1}$ in HCN-AMS medium (Fig. 5). It is interesting here to note that the EPS production increased by 4.2 to $75 \%$ in HCN-AMS medium. The highest EPS production of $0.41 \mathrm{mgmL}^{-1}$ was recorded in CBMB27 strain in HCN-AMS medium. Except for CBMB 110, increases in EPS production in all strains were statistically significant.
Qualitative PHB production Inclusions are aggregates of various compounds that are normally involved in storing energy reserves or building blocks for the cell. Inclusions accumulate when a cell is grown in the presence of excess nutrients and they are often observed under laboratory conditions. One of the more common storage inclusions is PHB. Phase contrast microscopy was used to detect brightly refractile cytoplasmic inclusions in PHB producing bacteria under axenic conditions. All Methylobacterium strains showed well-defined brightly refractile cytoplasmatic inclusions under Phase contrast microscopy (Fig. 6), suggesting PHB accumulation by these strains.

Quantitative PHB production Quantitative PHB production in Methylobacterium strains was compared using AMS medium and HCN-AMS medium based on spectroscopic assay. All the studied strains were found to be positive for PHB accumulation. Methylobacterium strains CBMB20, CBMB27, CBMB35, and CBMB110 recorded a PHB content of $0.56,1.15,0.83$, and $0.78 \mathrm{mg}$ $\mathrm{ml}^{-1}$ and $0.56,1.20,1.13$, and $0.87 \mathrm{mg} \mathrm{mL}^{-1}$ in HCN-AMS medium (Fig. 7). PHB production in Methylobacterium strains when grown in HCN-AMS medium increased by 3.8, 36.7, and $12.0 \%$ in CBMB27, CBMB37, and 

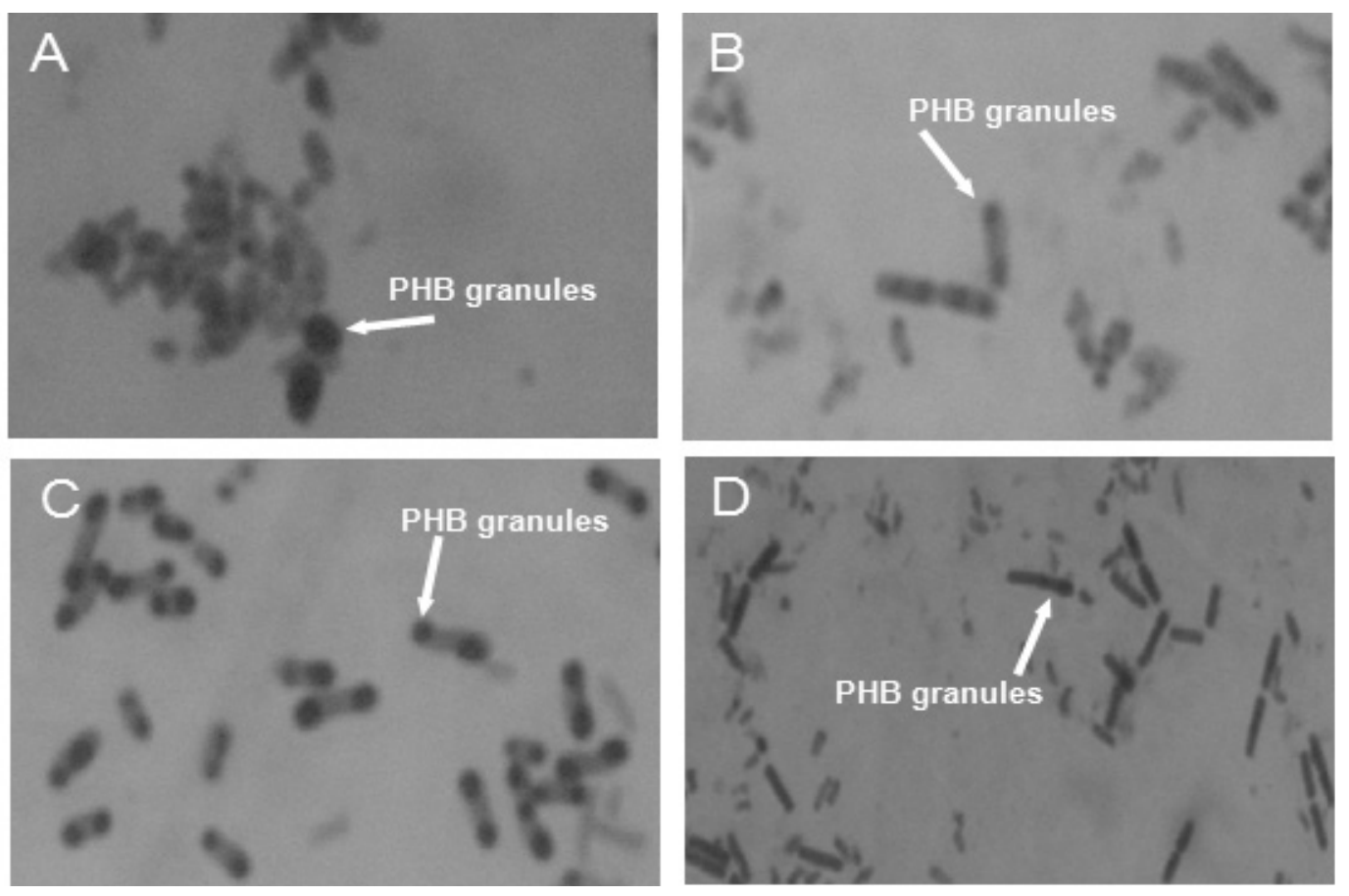

Fig. 6. Micrographs of Methylobacterium stains showing PHB accumulation by dark inclusions with sudan black stain. A) CBMB20; B) CBMB27; C) CBMB35; D) CBMB110.

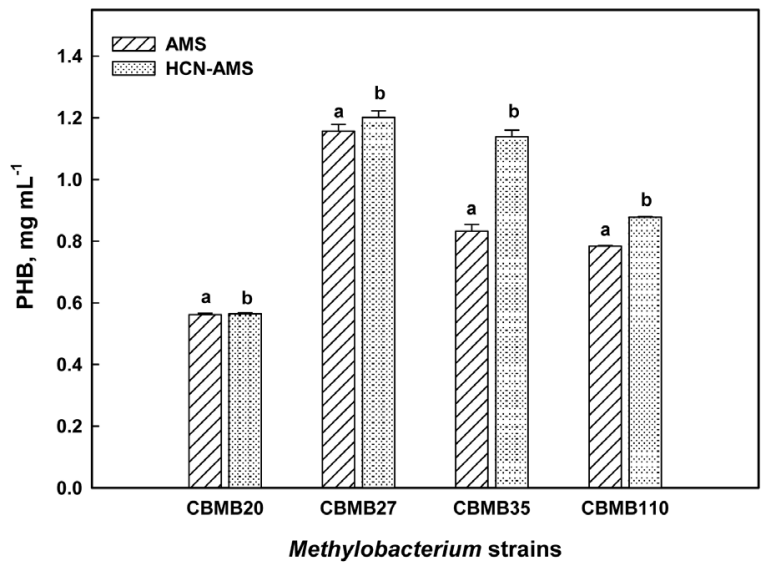

Fig. 7. PHB accumulation in Methylobacterium strains grown in AMS and HCN-AMS medium maintained at 150 rpm at $30^{\circ} \mathrm{C}$. Each value represents the mean $\pm \operatorname{S.E}(n=4)$. Different letters after values indicate a significant difference (among growth media) according to $t$ test at $p \leq 0.05$.

CBMB110 strains. The highest PHB accumulation of 1 to $1.20 \mathrm{mg} \mathrm{mL}^{-1}$ was recorded by the strain CBMB27 grown HCN-AMS medium.

\section{Stress endurance efficiency under different created stress conditions}

Osmotic stress Survivability of Methylobacterium strains grown in HCN-AMS medium and AMS medium was compared under $\mathrm{NaCl}$ stress conditions and the results are presented in Table 2. Bacterial populations decreased with increase in $\mathrm{NaCl}$ concentration regardless of Methylobacterium strains or the media used. Highest reduction in population was recorded at $250 \mathrm{mM} \mathrm{NaCl}$, at which CBMB20, CBMB27, CBMB35, and CBMB110 populations decreased by $5.3,3.2,1.6$, and $2.6 \%$, respectively in HCN-AMS medium and 6.0, 3.5, 7.3, and $7.9 \%$ in AMS medium. Decrease in populations grown in HCN-AMS medium was comparatively less for strains CBMB27, CBMB35, and CBMB110.

At $200 \mathrm{mM}$ concentration, notable reduction in bacterial population was observed for the strain CBMB110, with $2.8 \%$ decrease in HCN-AMS medium compared to $5.4 \%$ in AMS medium. Notable differences were also observed for the strains CBMB20, CBMB35, and CBMB110, with 2.3, 3.2, and 3.2\% decrease, respectively in HCN-AMS medium and 1.8, 1.7, and 2.7\% decrease, respectively in AMS medium at $150 \mathrm{mM} \mathrm{NaCl}$.

Acid stress Survivability of Methylobacterium strains grown in HCN-AMS medium was compared with normal AMS medium when subjected to acid stress. The results are presented in Table 3. Acid stress at $\mathrm{pH} 5.0$ caused 3.6, 5.9, 4.2, and 7.7\% decrease in population of CBMB20, CBMB27, CBMB35, and CBMB110 in AMS medium and $0.9,2.4,1.3$, and $4.1 \%$ decrease cor- 
Table 2. Osmotic stress tolerance among Methylobacterium strains grown in AMS medium and HCN-AMS medium.

\begin{tabular}{lccccccc}
\hline \hline \multirow{2}{*}{ Treatments } & \multirow{2}{*}{ Strains } & \multicolumn{3}{c}{ AMS } & \multicolumn{3}{c}{ HCN-AMS } \\
\cline { 3 - 8 } & & Before stress & After stress & Decrement & Before stress & After stress & Decrement \\
\hline \multirow{2}{*}{$150 \mathrm{mM}$} & CBMB20 & $6.07 \pm 0.04$ & $5.94 \pm 0.04$ & 2.30 & $6.28 \pm 0.03$ & $6.17 \pm 0.03$ & 1.83 \\
$\mathrm{NaCl}$ & CBMB27 & $5.92 \pm 0.06$ & $5.82 \pm 0.05$ & 1.72 & $6.37 \pm 0.03$ & $6.28 \pm 0.03$ & 1.38 \\
& CBMB35 & $6.18 \pm 0.05$ & $5.99 \pm 0.07$ & 3.27 & $6.33 \pm 0.03$ & $6.22 \pm 0.03$ & 1.75 \\
& CBMB110 & $6.24 \pm 0.05$ & $6.04 \pm 0.07$ & 3.25 & $6.26 \pm 0.02$ & $6.09 \pm 0.03$ & 2.73 \\
\hline \multirow{2}{*}{$200 \mathrm{mM}$} & CBMB20 & $6.11 \pm 0.05$ & $5.94 \pm 0.02$ & 2.93 & $6.32 \pm 0.08$ & $6.17 \pm 0.02$ & 2.39 \\
$\mathrm{NaCl}$ & CBMB27 & $6.11 \pm 0.06$ & $5.91 \pm 0.03$ & 3.33 & $6.29 \pm 0.02$ & $6.08 \pm 0.03$ & 3.58 \\
& CBMB35 & $6.07 \pm 0.05$ & $5.99 \pm 0.07$ & 1.25 & $6.31 \pm 0.03$ & $6.22 \pm 0.03$ & 1.50 \\
& CBMB110 & $6.23 \pm 0.06$ & $5.91 \pm 0.07$ & 5.40 & $6.18 \pm 0.02$ & $6.01 \pm 0.03$ & 2.89 \\
\hline \multirow{2}{*}{$250 \mathrm{mM}$} & CBMB20 & $5.99 \pm 0.03$ & $5.64 \pm 0.10$ & 6.04 & $6.00 \pm 0.06$ & $5.69 \pm 0.04$ & 5.33 \\
$\mathrm{NaCl}$ & CBMB27 & $5.98 \pm 0.05$ & $5.78 \pm 0.08$ & 3.52 & $5.99 \pm 0.09$ & $5.81 \pm 0.08$ & 3.21 \\
& CBMB35 & $6.11 \pm 0.04$ & $5.69 \pm 0.05$ & 7.36 & $6.31 \pm 0.3$ & $6.21 \pm 0.02$ & 1.68 \\
& CBMB110 & $5.90 \pm 0.04$ & $5.46 \pm 0.06$ & 7.94 & $6.17 \pm 0.05$ & $6.01 \pm 0.03$ & 2.67 \\
\hline
\end{tabular}

*Each value represents the mean \pm S.E $(n=4)$.

Table 3. Acid stress tolerance among Methylobacterium strains grown in AMS medium and HCN-AMS medium.

\begin{tabular}{|c|c|c|c|c|c|c|c|}
\hline & \multirow{2}{*}{ Strains } & \multicolumn{3}{|c|}{ AMS } & \multicolumn{3}{|c|}{ HCN-AMS } \\
\hline & & Before stress & After stress & Decrement & Before stress & After stress & Decrement \\
\hline \multirow{5}{*}{$\mathrm{pH} 5.0$} & & \multicolumn{2}{|c|}{--- $\log$ cfu $\mathrm{mL}^{-1}---$} & $\%$ & \multicolumn{2}{|c|}{--- $\log$ cfu $\mathrm{mL}^{-1}---$} & $\%$ \\
\hline & CBMB20 & $5.99 \pm 0.03$ & $5.77 \pm 0.04$ & 3.69 & $6.22 \pm 0.02$ & $6.16 \pm 0.05$ & 0.99 \\
\hline & CBMB27 & $5.79 \pm 0.05$ & $5.46 \pm 0.06$ & 5.90 & $6.25 \pm 0.05$ & $6.11 \pm 0.07$ & 2.41 \\
\hline & CBMB35 & $6.07 \pm 0.06$ & $5.82 \pm 0.09$ & 4.25 & $5.84 \pm 0.03$ & $5.77 \pm 0.06$ & 1.34 \\
\hline & CBMB110 & $5.84 \pm 0.04$ & $5.42 \pm 0.07$ & 7.72 & $6.23 \pm 0.05$ & $5.98 \pm 0.05$ & 4.15 \\
\hline
\end{tabular}

*Each value represents the mean \pm S.E $(n=4)$.

respondingly in HCN-AMS medium. Percentage decrease of Methylobacterium populations in HCN-AMS medium was likewise, lower when compared to AMS medium.

\section{DISCUSSION}

The increase in the world's population, coupled with the limitations in the world's supply of natural resources and widespread degeneration of the environment presents a major challenge to agriculturists. Though chemical fertilizers can meet the nutrient requirements of the plant within a short period, excessive use had caused them to be washed away and contaminate water leading to evaporation in the atmosphere and environmental pollution.

Microbial inoculants, also known as soil inoculants are agricultural amendments that use beneficial microbes (bacteria or fungi) to promote plant health. Microbial inoculants are applied to improve plant nutrition and can also be used to promote plant growth by stimulating plant hormone production (Bashan and Holguin, 1997; Sullivan et al, 2001). Groups of bacteria, including strains from genera Azospirillium, Azotobacter, Bacillus, Burkholderia, Erwinia, Methylobacterium, Pseudomonas, Rhizobium, and Serratia have gained acceptance as plant growth promoting rhizobacteria (PGPR).

Members of the genus Methylobacterium are adaptable in nature and ubiquitous on plant surfaces, with high potential for predominating the phyllosphere (Corpe and Rheem, 1989). Methylobacterium, as plant symbionts, utilize the methanol emitted from plants and in turn, impart beneficial effects on plant growth through one or more mechanisms that include production of phytohormones like indoleacetic acid or cytokinins and vitamins (Basile et al., 1985; Koenig et al., 2002; 
Trotsenko et al., 2001), synthesis of enzymes such as urease or 1-aminocyclopropane-1-carboxylate deaminase (ACCD) that modulate plant growth (Holland and Polacco, 1992; Madhaiyan et al., 2006, 2007) and production of siderophores (Idris et al., 2004).

Monier and Lindow (2003) reported that on different plant species, large aggregates of bacteria are embedded in extracellular matrix. Further studies by Schauer and Kutschera (2008) reported that leaf associated Methylobacterium live in plant surfaces in the form of biofilm like aggregates and suggested the need to further work to support this hypothesis. Aggregation in bacterial strains such as Azospirillum, Azotobacter and Pseudomonas positively affected its dispersal in soil (Madi and Henis, 1989). In this study, we investigated aggregation in Methylobacterium strains grown under high carbon concentrations, and the influence of such modification to EPS production, PHB accumulation and stress tolerance efficiency.

Aggregation was studied using Methylobacterium strains grown in HCN-AMS medium. The strains CBMB35 and CBMB27 recorded the highest floc yield of 8.5 and $7.25 \mathrm{mg} \mathrm{mL}^{-1}$ dry weight in HCN-AMS medium. Numerous studies have shown that rich media with a low $\mathrm{C}: \mathrm{N}$ ratio tend to promote a dispersive growth mode, whereas a medium with high $\mathrm{C}: \mathrm{N}$ ratio tend to aggregate and flocculate the bacterial cells (Burdman et al., 1998; Madi and Henis, 1989; Sadasivan and Neyra, 1985). Furthermore, fructose plays a major role in aggregation by enhancing polysaccharide synthesis (Kaci et al., 2005; Sadasivan and Neyra, 1985) and this polysaccharide induce flocculation by bridging of polymers between the cells (Del Gallo et al., 1989).

All Methylobacterium strains were found to be positive for Calcoflour staining as evidenced by the positive fluorescence. Calcofluor-white is a special fluorescent stain that binds strongly to structures containing cellulose present in the polysaccharides (Dell Gallo et al., 1989). The positive staining with Calcoflour reveal the presence of calcofluor-binding polysaccharides distributed between the capsular and exopolysaccharide fractions in the Methylobacterium strains.

The total EPS content of Methylobacterium strains grown in HCN-AMS medium was analyzed and the results show that the EPS content among the selected plant growth promoting Methylobacterium strains varied from 0.24 to $0.34 \mathrm{mg} \mathrm{mL}^{-1}$ in AMS medium and from 0.24 to
$0.41 \mathrm{mg} \mathrm{mL}^{-1}$ in HCN-AMS medium. Breuer and Babel (1999) reported the production of $3.86 \mathrm{~g} \mathrm{~L}^{-1}$ of EPS in $M$. rhodesiunum under ammonium limitation conditions. Our results correspond with findings for EPS production under conditions of nitrogen limitation. Data suggesting the involvement of EPS in aggregation of numerous PGPR strains have been published earlier (Alami et al., 2000; Bahat-samet et al., 2004; Burdman et al., 2000). Similar findings could also be observed in our present study in which strains CBMB20, CBMB27, and CBMB35, showing better EPS production also yielded higher flocs.

All Methylobacterium strains showed well-defined brightly refractile cytoplasmatic inclusions under Phase contrast microscopy suggesting PHB accumulation by these strains. The presence of brightly refractile cytoplasmic inclusions in PHB producing bacteria under axenic conditions and the subsequent confirmation of PHB production as observed in our present study go well with the findings of López-Cortés et al. (2008), who used the presence of bright cytoplasmic inclusions as preliminary step for qualitative PHB determination. Micrographs of Methylobacterium stains showed positive for PHB accumulation as evidenced by the dark inclusions with Sudan black stain. The presence of these dark inclusions after sudan black staining may be explained based on non fluorescent lipophilic affinity of the stain towards PHB (Wei et al., 2011).

An increase in PHB accumulation was observed in Methylobacterium strains grown in HCN-AMS medium. The high amount of PHB accumulation observed in Methylobacterium strains was also recorded by Alvarez et al. (1996) in some psychrophilic and psychrotrophic crude oil-utilizing marine bacteria, which accumulate lipid storage compounds in the cytoplasm under nitrogenlimiting conditions when the $\mathrm{C}: \mathrm{N}$ ratio becomes high.

Better survivability was observed in Methylobacterium strains grown in HCN-AMS medium when subjected to osmotic and acid stress. Though reports regarding the survivability of Methylobacterium strains subjected to different stress responses are scarce, we believe that the enhanced survivability of Methylobacterium strains may be attributed to the high EPS production and PHB production under increased carbon concentration in the growth medium. The role of high carbon concentration in aggregation of bacterial strains such as Azospirillum, Azotobacter, and Pseudomonas and the concomitant accumulation of high levels of intracellular storage 
substances such as polyhydroxyalkanoates (PHA) has been reported by numerous workers (Burdman et al., 1998; Burdman et al., 2000; Kadouri et al., 2003; Madi and Henis, 1989). Results of our present study go well with the findings of Ayub et al. (2004), who correlated high stress tolerance efficiency in Pseudomonas sp. 14-3 for thermal and oxidative stresses to the high PHB accumulation by the strain.

\section{Conclusion}

In this study, we investigated aggregation in Methylobacterium strains grown under high carbon concentrations, and the influence of such modification to EPS production, PHB accumulation and stress tolerance efficiency. Our present study in which strains CBMB20, CBMB27, and CBMB35, which showed better EPS production also yielded higher flocs. An increase in PHB accumulation was observed in Methylobacterium strains grown in HCN-AMS medium. Better survivability was observed in Methylobacterium strains grown in HCNAMS medium when subjected to osmotic and acid stress. Though reports regarding the survivability of Methylobacterium strains subjected to different stress responses are scarce, we believe that the enhanced survivability of Methylobacterium strains may be attributed to the high EPS production and PHB production under increased carbon concentration in the growth medium.

\section{Acknowledgements}

This work was partially supported by the Rural Development Administration (RDA), Republic of Korea.

\section{References}

Alami, Y., W. Achouak, C. Marol, and T. Heulin. 2000. Rhizosphere soil aggregation and plant growth promotion of sunflowers by an exopolysaccharide-producing Rhizobium sp. strain isolated from sunflower roots. Appl. Environ. Microbiol. 66:3393-3398.

Alvarez, M.I., R.J. Sueldo, and C.A. Barassi. 1996. Effect of Azospirillum inoculation on coleoptile growth in wheat seedlings under water stress. Cereal Res. Commun. 24:101-107.

Ayub Akbari, P.J. Swedko, H.D. Clark, W. Hogg, J. Lemelin, P. Magner, L. Moore, and D. Ooi. 2004. Detection of chronic kidney disease with laboratory reporting of estimated glomerular filtration rate and an educational program. Arch.
Intern. Med. 164(16):1788-1792

Bahat-Samet, E., S. Castro-Sowinski. and Y. Okon. 2004. Arabinose content of extracellular polysaccharide plays a role in cell aggregation of Azospirrilum brasilense. FEMS Microbial. Lett. 237: 195-203

Bashan, Y. and G. Holguin. 1997. Azospirillum - plant relationships: environment and physiological advances. Can. J. Microbiol. 43:103-121.

Bashan, Y. 1998. Inoculants of plant growth-promoting bacteria for use in agriculture. Biotechnol. Adv. 16:729-770.

Basile, D.V., M.R. Basile, Q.Y. Li, and W.A. Corpe. 1985. Vitamin B12-stimulated growth and development of Jungermannia leiantha Grolle and Gymnocolea inflata (Huds.) Dum (Hepaticae). Bryologist. 88:77-81.

Breuer, U. and W. Babel. 1999. Methylobacterium rhodesianum produces poly-3-hydroxybutyrate and after mutagenesis in addition exopolysaccharides. Acta Biotechnol. 19:779-786.

Burdman, S., E. Jurkevitch, B. Schnartsburd, M. Hampel, and Y. Okon. 1998. Aggregation in Azospirillum brasilense: effects of chemical and physical factors and involvement of extracellular components. Microbiol. 144:1989-1999

Burdman, S., E. Jurkevitch, M.E. Soria-Dia, A.M.G. Serrano, and Y. Okon. 2000. Extracellular polysaccharide composition of Azospirillum brasilense and its relation to cell aggregation. FEMS Microbiol. Lett. 189:259-264.

Catroux, G., A. Hartmann, C. Revellin. 2001. Trends in rhizobial inoculant production and use. Plant Soil 230:21-30.

Celik, G.Y., B. Aslim, and Y. Beyatli. 2008. Characterization and production of the exopolysaccharide (EPS) from Pseudomonas aeruginosa G1 and Pseudomonas putida G12 strains. Cabohydrate Polymers. 73:178-182.

Choi, H.H. 1998. An explicit formula of linear sliding surfaces for a class of uncertain dynamic systems with mismatched uncertainties. Automatica 34:1015-1020.

Corpe, W.A. 1985. A method for detecting methylotrophic bacteria on solid surfaces. J. Microbiol. Methods 3:215-221.

Corpe, W.A. and S. Rheem. 1989. Ecology of the methylotrophic bacteria on living leaf surfaces. FEMS Microbiol. Ecol. 62:243-250

Dana, J.R., and L.J. Shimkets. 1993. Regulation of cohesion dependent cell interactions in Myxococcus xanthus. J. Bacteriol. 175:3636-3647.

Del Gallo M, M. Negi, and C.A. Neyra. 1989. Calcofluor- and lectin-binding exocellular polysaccharides of Azospirillum brasilense and Azospirillum lipoferum. J. Bacteriol. vol. 171 no. 6:3504-3510.

Gallego, V., M.T. Garcia, and A. Ventosa, 2006. Methylobacterium adhaesivum sp. nov., a methylotrophic bacterium isolated from drinking water. Int. J. Syst. Evol. Microbiol. 56:339-342.

Gomez, K.A. and A.A. Gomez. 1984. Statistical procedures for agricultural research, 2nd ed. (Chichester, UK: John Wiley and Sons).

Green, P. N. 2001. Methylobacterium. In The Prokaryotes, 3rd edn, release 3.5. Edited by M. Dworkin. New York: Springer.

Holland M.A. and J.C. Polacco. 1994. PPFMs and other contaminants: is there more to plant physiology than just plant 
Annu. Rev. Plant Physiol. Plant Mol. Biol. 45:197-209.

Idris, R., R. Trifonova, M. Puschenreiter, W.W. Wenzel, and A. Sessitsch. 2004. Bacterial communities associated with flowering plants of the $\mathrm{Ni}$ hyper accumulator Thaspi goesingense. Appl. Environ. Microbiol. 70:2667-2677.

Kaci, Y., A. Heyraud, M. Barakat, and T. Heulin. 2005. Isolation and identification of an EPS producing Rhizobium strain from arid soil (Algeria): characterization of its EPS and the effect of inoculation on wheat rhizosphere soil structure. Res. Microbiol. 156:522-531.

Kadouri, D., E. Jurkevitch, and Y. Okon. 2003. Involvement of the reserve material poly-beta-hydroxybutyrate in Azospirillum brasilense stress endurance and root colonization. Appl. Environ. Microbiol. 69:3246-3253.

Karr, D.B., J.K.Waters and D. W. Emerich. 1983. Analysis of poly-ß-hydroxybutyrate in Rhizobium japonicum bacteroids by ion-exclusion high-pressure liquid chromatography and UV detection. Appl. Environ. Microbial. 46, 1339- 1344.

Koenig, R.L., Morris, R.O. and Polacco, J.C. 2002. tRNA is the source of low-level trans-zeatin production in Methylobacterium spp.. J Bacteriol. 184: 1832-1842.

Knief, C., L. Frances, F. Cantet, and J.A. Vorholt. 2008. Cultivation-independent characterization of Methylobacterium populations in the plant phyllosphere by automated ribosomal intergenic spacer analysis. Appl. Environ. Microbiol. 74:2218-2228.

López-Cortés, A., A. Lanz-Landázuri, and J.Q. GarcíaMaldonado. 2008. Screening and isolation of PHB-producing bacteria in a polluted marine microbial mat. Microb. Ecol. 56:112-120.

Madhaiyan, M., B.Y. Kim, S. Poonguzhali, S.W. Kwon, M.H. Song, J.H. Ryu, S.J. Go, B.S. Koo, and T.M. Sa. 2007. Methylobacterium oryzae sp. nov., an aerobic, pink-pigmented, facultatively,ethylotrophic, 1-aminocyclopropane-1-carboxylate deaminase-producing bacterium isolated from rice. Int. J. Syst. Evol. Microbiol. 57:326-331.

Madhaiyan, M., S. Poonguzhali, V.S. Saravanan, K. Hari, and T.M. Sa. 2006. Influence of pesticides on the growth rate and plant-growth promoting traits of Gluconacetobacter diazotrophicus. Pestic. Biochem. Physiol. 84:143-154.

Madi, L. and Y. Henis. 1989. Aggregation in Azospirillum brasilense cd.: Conditions and factors involved in cell-to-cell adhesion. Plant Soil 115:89-98.

Monier, J.M. and S.E. Lindow. 2003. Differential survival of solitary and aggregated bacterial cells promotes aggregate formation on leaf surfaces. Proc Natl Acad Sci USA. 100(26): 15977-15982.

Neyra, C.A., L.A. Atkinson, and O. Olubayi. 1995. Coaggregation of Azospirillum with other bacteria: basis for functional diversity. In: Fendrik I, Del Gallo M, Vanderleyden J, de Zamaroczy M (eds) Azospirillum VI and related microorganisms, genetics physiology ecology. NATO ASI series, series G. springer. Berlin Heidelberg New York. 429-439.

Omer, Z.S., R. Tombolini, A. Broberg, and B. Gerhardson. 2004. Indole-3-acetic acid production by pink-pigmented facultative methylotrophic bacteria. Plant Growth Regul. 43:93-96.

Ramaswamy, S., M. Dworkin, and J. Downard. 1997. Identification and characterization of Myxococcus xanthus mutants deficient in calcofluor white binding. J. Bacteriol. 179:2863-2871.

Reusch, R.N. and H.L. Sadoff. 1983. D-(-) Poly-ß-hydroxybutyrate in membranes of genetically competent bacteria. J. Bacteriol. 156:778-788.

Sadasivan, L. and C.A. Neyra. 1985. Flocculation in Azospirillum brasilense and Azospirillum lipoferum: exopolysaccharides and cyst formation. J. Bacterial. 163:716-723.

Schauer, S. and U. Kutschera. 2008. Methylotrophic bacteria on the surfaces of field-grown sunflower plants: a biogeographic perspective. Theory Biosci.. 127:23-29.

Schauer S., P. Kämpfer, S. Wellner, C. Spröer, and U. Kutschera. 2011. Methylobacterium marchantiae sp. nov. a pinkpigmented, facultatively methylotrophic bacterium isolated from the thallus of a liverwort. Int. J. Syst. Evol. Microbiol. 61:870-876.

Sullivan. B.D., M.R. Dana, and D.A. Sullivan. 2001. Influence of aging on the polar and neutral lipid profiles in human meibomian gland secretions. Invest. Ophthalmol. Vis. Sci.. 124(9):1286-1292.

Trotsenko, Y.A., Ivanova, E.G. and Doronina, N.V. 2001. Aerobic methylotrophic bacteria as phytosymbionts. Mikrobiologiya. 70: 725-736.

Van Elsas, J.D. and C.E. Heijnen. 1990. Methods for the introduction of bacteria in soil: a review. Biol. Fertil. Soils 10:127-33.

Wei Y.H, W.C. Chen, C.K. Huang, H.S. Wu, Y.M. Sun, C.W. Lo, and O.M. Janarthanan. 2011. Screening and Evaluation of Polyhydroxybutyrate-Producing Strains from Indigenous Isolate Cupriavidus taiwanensis Strains. Int. J. Mol. Sci.. 12:252-265. 